\title{
AUTOLESIONES NO SUICIDAS Y SU RELACIÓN CON EL ESTILO DE APEGO EN UNA MUESTRA NO CLÍNICA DE ADOLESCENTES CHILENOS
}

NON-SUICIDAL SELF-HARM AND THEIR RELATIONSHIP WITH ATTACHMENT STYLE IN A NON-CLINICAL SAMPLE OF CHILEAN ADOLESCENTS

Recibido: 13 de diciembre de 2018 | Aceptado: 13 de marzo de 2019

\author{
JORGE EDUARDO LEIVA PEREIRA 1 , ANA MATILDE CONCHA LANDEROS 2
}

1., 2. UNIVERSIDAD DE SANTIAGO DE CHILE, Santiago de Chile, Chile

\begin{abstract}
Objective: to establish the relationship between the presence of non-suicidal self-harm with the type of attachment in a sample of Chilean adolescents. Method: We used a questionnaire to evaluate non-suicidal selfharm and two questionnaires to measure attachment style. In this non-experimental, cross-sectional and analytical study, we used Chi-square test for comparisons of attachment styles between participants who selfharm and those who do not. 186 adolescents participated, with ages between 14 and 18 years old $(M=15.8$, SD $=1.1), 57 \%$ correspond to women. Results: $11 \%$ (22) of the subjects reported self-harm, $77.3 \%$ corresponded to women. In subjects who reported self-harm, $80.9 \%$ had an unsafe type of attachment (4.8\% avoidant, $33.3 \%$ ambivalent and $42.8 \%$ disorganized). $95.4 \%$ have medium to high scores in the subscales of anger and anguish towards the figure of attachment. Conclusions: Insecure and disorganized attachment styles are a factor of vulnerability that predisposes subjects to emotional deregulation. Adolescents who report selfharm tend to have an insecure attachment, in contrast to those who do not self-harm.
\end{abstract}

KEY WORDS: Non-suicidal self-harm, attachment, adolescents.

\section{CÓMO CITAR / HOW TO CITE}

Leiva Pereira, J. E. \& Concha Landeros, A. M. (2019). Autolesiones no suicidas y su relación con el estilo de apego en una muestra no clínica de adolescentes chilenos. Salud \& Sociedad, 10(1), 84-99. doi: $10.22199 / \mathrm{S} 07187475.2019 .0001 .00006$

\footnotetext{
L. Psicólogo Magister en Terapia Post- Racionalista, Centro de Salud Mental Comunitaria, Servicio de Salud de Maule, Talca, Chile código postal 3471076, Chile. 0RCID: 0000-00034439-5836. E-mail: j.leiva.pereira@gmail.com

2. Médico Psiquiatra de niños y adole scentes, Centro de Salud Mental Comunitaria, Servicio de Salud de Maule, Talca, Chile código postal 3471076 \& Facultad de Medicina, Univer sidad Católica del Maule, Chile, código postal 3480112. E-mail: anamatconcha@gmail.com
} 
Los adolescentes son un grupo muy proclive a presentar conductas de riesgo, tales como altos índices de consumo de alcohol, tabaco y otras sustancias psicoactivas; actividad sexual de riesgo a edades cada vez más tempranas; elementos que se asocian a la búsqueda de identidad y crisis relacionadas a cambios hormonales y conductuales; lo que se relaciona de una u otra forma con embarazos no deseados, infecciones de transmisión sexual y un elevado riesgo de traumatismos, tanto intencionados como accidentales (OMS, 2015).

Dentro de las conductas de riesgo numerosos autores (Hawton, Rodham, Evans, y Weatherall, 2002; Nock, Joiner, Gordon, Lloyd-Richardson, y Prinstein, 2006; Ougrin y Boege, 2013) han relevado el aumento de las autolesiones en este grupo etario, las cuales se encuentran en diversos cuadros psiquiátricos dada su naturaleza compleja y multifactorial y su alta fluctuación en breves períodos de tiempo (Ougrin $Y$ Boege, 2013; Villarroel et al., 2013), así como su alta relación con el suicidio, aumentando el riesgo en hasta 10 veces (Hawton y Harriss, 2007), pues incluso se ha considerado que las autolesiones no suicidas son un factor de riesgo principal para el suicidio, especialmente en pacientes con trastornos de salud mental y aquellos que han estado hospitalizados, independiente del sexo, edad y el método de autolesión (Pompili et al., 2015). De ahí, la importancia de indagar su presencia al momento de evaluar adolescentes con conducta suicida pues, aunque las autolesiones no suicidas como su nombre lo plantea no implica ideación suicida, si se transforman en un factor de riesgo relevante.

En las últimas décadas las autolesiones han cobrado interés científico, es así, que se estima en algunos estudios en población no clínica, una prevalencia en la vida de 7,5-8 \% en pre-adolescentes, llegando a un 12-28 \% en adolescentes, y hasta un 40-80 \% en adolescentes de muestras clínicas (Frías Ibáñez, Vázquez Costa, Del Real Peña,
Sánchez del Castillo, y Giné Servén, 2012; Washburn et al., 2012). La edad de inicio se sitúa entre los 11 y 15 años, con una media de 12,5 años (Nixon, Cloutier, y Jansson, 2008).

Los estudios reflejan que las autolesiones se presentan más en mujeres que en hombres (Hawton et al., 2002; Patton et al., 2007; Morey et al., 2008; O'Connor et al., 2009; Shin et., 2009; Landstedt y Gillander, 2011, en Frías Ibáñez et al., 2012), lo cual es corroborado en una revisión sistemática en torno al curso longitudinal de las autolesiones no suicidas y el auto-daño deliberado (Plener, Schumacher, Munz, y Groschwitz, 2015); aunque algunas comparaciones en muestras no clínicas no han encontrado diferencias en cuanto a género (Kirchner et al., 2011; Wan et al., 2011 en Frías Ibáñez et al., 2012), y otros estudios refieren diferencias en el método empleado (Lundh, Karim y Quilisch, 2007; Laukkanen, Rissanen, Honka - lampi, Kylma, Tolmunen y Hintikka, 2009 en Frías Ibáñez et al., 2012), con un predominio de autolesiones a través de cortes en mujeres y quemaduras en hombres (Cerutti, Manca, Pre - saghi y Gratz 2011 en Frías Ibáñez et al., 2012).

Las principales líneas de investigación sobre autolesiones corresponden a estudios de índole psiquiátrica centrados en aspectos epidemiológicos, factores protectores y de riesgo, así como la comorbilidad con psicopatología específica, incluso algunos plantean la idea de que podría ser un síntoma de algún trastorno, además de ser un factor de riesgo para el suicidio (Favazza, 1996; Gratz, 2003; Rojas, 2016). Aunque también otros teóricos lo plantean como un síndrome específico (Nader y Morales, 2011).

Nuevas investigaciones en autolesiones no suicidas en adolescentes plantean que dichas autolesiones responden a factores intrapersonales (manejo del estrés, regulación emocional, manejo de angustia 
psicológica), así como a factores interpersonales (estilo de crianza, apego, influencia de los pares) (American Psychiatric Association, 2014; Baetens et al., 2014; Hollander, 2017).

Además de ser consideradas como un precursor del desarrollo de trastorno límite de personalidad, en el contexto de experiencias adversas en la infancia en el plano interpersonal (negligencia emocional, abuso sexual, etc.) (Hessels, Laceulle, van Aken, Resch, y Kaess, 2018). En el presente estudio consideramos las autolesiones más frecuentes, descritas en la literatura, sin diferenciación entre el tipo de autolesión (cortar, quemar, pinchar, golpear, etc.).

Desde ahí el interés por revisar este tipo de autolesiones y los patrones de apego, ya que siendo la adolescencia un período de transición, donde el adolescente comienza un tremendo esfuerzo para volverse menos dependiente de las figuras de apego primario (Eg. los padres) (Balabanian, Lemos, y Vargas, 2014) se ve enfrentado a profundas transformaciones en los sistemas emocionales, cognitivos y conductuales que rodean las relaciones de apego.

Existe evidencia (Crowell, Beauchaine, y Linehan, 2009; Gandhi, et al., 2019) de que aquellos sujetos con sentidos de vinculación perturbado pueden llegar a utilizar estrategias autodestructivas como las ANS para la regulación del afecto como forma de compensar la falta percibida de apoyo interpersonal. Los estilos de apego en este periodo cobran un protagonismo especial, ya que es uno de caracter evolutivo en el que se establecen vínculos intensos en el plano de la amistad y en las relaciones de pareja. A su vez se incrementa la capacidad reflexiva sobre uno mismo y los demás, lo que favorece que se revisen los vínculos establecidos con los progenitores o figuras sustitutivas (Allen, McElhaney, Kuperminc y Jodl, 2004; Allen, Moore, Kuperminc y Bell, 1998; Sánchez-Queija y Oliva, 2003 en Camps-Pons, Castillo-Garayoa, y Cifre, 2014).

\section{Autolesiones}

Es común encontrar referencia a las autolesiones en muchos contextos, ya sea religiosos, como ritos antropológicos, contextos artísticos y literarios (Favazza, 1996), incluso en medios actuales tanto en televisión, cine, música e internet (Nock, 2010).Las autolesiones se podrían definir como todo comportamiento que se realiza intencionalmente a sí mismo, y con el conocimiento de que pueden resultar en cierto grado de daño físico o psicológico, existiendo una distinción entre aquellos comportamientos en los que la lesión corporal es el propósito, y aquellos en los que es un subproducto involuntario (Nock, 2010).

Es relevante mencionar que existen dos visiones respecto a la temática de las autolesiones, por un lado, los grupos de investigación europeos plantean una definición meramente conductual, independiente de la motivación detrás de la conducta, utilizándose el término autolesión deliberada (deliberate self-harm). La autolesión deliberada ha sido definida como todo acto que realiza un individuo de manera intencional contra sí mismo para hacerse daño (Eg. cortes, quemaduras, sobreingestas medicamentosas y sobredosis, envenenamiento, golpes, saltar desde lo alto de un lugar, etc.) cuyo resultado no es fatal, y que puede ser sancionable por la cultura (Frías Ibáñez et al., 2012; Hawton et al., 2002).

Por otro lado, existe una línea de investigación, principalmente norteamericana, que define a las autolesiones como aquellas conductas que impliquen un daño directo y deliberado contra uno mismo, en ausencia de la intencionalidad suicida, utilizándose el término autolesión no suicida (non suicidal self-injury) (Baetens et al., 2014; Frías Ibáñez et al., 2012; Nock, 2010). 


\section{Autolesiones no suicidas}

Las autolesiones no suicidas están definidas como el acto deliberado de causarse daño a uno mismo mediante cortes, quemaduras, mutilación $u$ otros métodos traumáticos (Nock et al., 2006), en la cual se excluyen las ingestas de medicamentos y los envenenamientos con intención suicida (Mollá, Batlle, Treen, López, Sanz, Martín, Pérez, Bulbena, 2015). También se consideran las autolesiones como estrategia para calmar el dolor emocional; para el control del dolor extremo y experiencias aterradoras de emociones abrumadoras; para calmarse o sentir "algo" y como autocastigo (Hollander, 2017).

El Manual Diagnóstico y Estadístico de los Trastornos Mentales en su 5ta edición (DSM5) considera una nueva categoría asociada a aquellas afecciones que necesitan más estudios, y se encuentra en discusión para ser incluidas en el futuro como trastorno. Las autolesiones no suicidas están incluidas, y su característica principal es que la persona se infringe lesiones superficiales, aunque dolorosas, en la superficie de su propio cuerpo de una manera repetida, con el objetivo de reducir emociones negativas como la tensión, ansiedad, exceso de culpa, - resolver alguna dificultad interpersonal (American Psychiatric Association, 2014; Hollander, 2017).

Frente a la consulta sobre las consecuencias de esta conducta generalmente el individuo refiere una sensación de alivio inmediato, incluso pudiéndose asociar a un patrón de comportamiento de tipo adictivo cuando éste aparece con frecuencia asociado a urgencia $y$ ansias de realizarlo. Las heridas infligidas pueden hacerse más profundas y numerosas (American Psychiatric Association, 2014; Mollá et al., 2015).

Los métodos más frecuentes para hacerse daño son; cortarse con cuchillo, aguja, u otro objeto afilado, y las zonas corporales más comunes, son la cara dorsal del antebrazo y la región frontal anterior de los muslos. Es común observar una serie de cortes superficiales paralelos separados por 1 o 2 centímetros en una localización visible o accesible. Los cortes resultantes a menudo sangrarán y finalmente dejarán un patrón de cicatrices característico (American Psychiatric Association, 2014). Otros métodos utilizados son clavar una aguja o la punta de un cuchillo afilado en una determinada zona, realizar una quemadura superficial con una colilla de cigarro encendida, o quemar la piel frotándola de manera repetida (American Psychiatric Association, 2014).

La gran mayoría de individuos que se producen autolesiones no suicidas no buscan asistencia clínica, y la prevalencia en muestras no clínicas es del 13 - 28\% (Mollá et al., 2015), lo cual lo sitúa como un problema importante que muchas veces puede pasar desapercibido.

\section{Apego}

La teoría del apego (Bowlby, 1976, 1979, 1995) se concibe como la tendencia de los humanos a generar vínculos afectivos sólidos con determinadas personas a través de la vida. Constituyéndose en la actualidad como una de las teorías más influyentes en el campo de la psicología y las neurociencias (Oliva, 2004; Schore, 1997, 2001).

A partir de evidencia empírica se ha demostrado que el apego gestado en la primera infancia tiene gran incidencia en la forma en que se crean vínculos con otras personas en la edad adulta, influyendo en las relaciones interpersonales significativas, así como en su bienestar 0 ajuste socioemocional (Bowlby, 1976, 1979).

La teoría del apego toma en cuenta la tendencia a formar íntimos lazos emocionales con personas específicas; es parte de un componente básico de los seres humanos persistente a lo largo de la vida adulta, hasta la vejez; en la infancia dichos lazos se desarrollan con el cuidador SALUD \& SOCIEDAD | V. 10 | No. 1 | ENERO - ABRIL | 2019 
principal, que es generalmente la madre, al que se acude en busca de cuidado, consuelo y apoyo; y durante la adolescencia y la adultez, estos lazos se mantienen, pero son complementados con nuevos vínculos (Oliva, 2004).

Las experiencias de los infantes con sus cuidadores principales poseen un rol fundamental a la hora de entender las capacidades de establecer vínculos afectivos. Así Bowlby (1995), conceptualiza el "internal working model" o modelo interno operante, definido como una representación mental de sí mismo y de las relaciones con los otros, que permiten anticipar, interpretar y responder a la conducta de sus figuras de apego, integrando experiencias presentes y pasadas en esquemas cognitivos y emocionales, a partir de las relaciones con las figuras de apego. Elemento fundamental para entender el apego en adultos (West, Rose, Spreng, Sheldon-Keller, y Adam, 1998).

Los sujetos construyen modelos internos operantes (MIO) del mundo y de sí mismos, los MIO del mundo contribuyen a identificar quienes son las figuras de apego, donde se pueden encontrar y cómo pueden responder; así los MIO de sí mismo poseen como característica principal la de concebir cuán aceptable o inaceptable se es ante la figura de apego (Bowlby, 1976).

\section{Tipos de apego}

A partir de las situaciones experimentales y conceptualizaciones de Mary Ainsworth en los años '70 (Crittenden, 2006; Lecannelier, 2009; Oliva, 2004), se establecen tres patrones conductuales que eran representativos de los distintos tipos de apego: apego seguro, apego inseguro evitativo. apego inseguro ambivalente, apego desorganizado (Kristic, 2014; Lecannelier, 2009; Oliva, 2004; Main y Solomon, 1990).

\section{Apego Adolescente}

Existe evidencia para plantear que las relaciones y estilos de apego gestados en la infancia impactan todo el ciclo vital del ser humano, desde su adolescencia hasta su adultez, pues el vínculo que se establece entre niños y cuidadores predice la calidad del desarrollo intrapersonal e interpersonal (Balabanian et al., 2014; Feeney y Noller, 2001; Guzmán y Contreras, 2012; Oros, Vargas, y Krumm, 2014).

El apego influye durante todo el ciclo vital, mediante la progresiva internalización de la relación como modelo interno operante. De esta forma diferentes experiencias en el vínculo primario, se reflejarían en diferentes expectativas acerca de la disponibilidad del cuidador, el sí mismo y la forma de ver las relaciones sociales y el mundo, siendo los estilos de apego construidos en la infancia en gran medida un predictor sobre los procesos vinculares posteriores (Balabanian et al., 2014; Feeney y Noller, 2001; Guzmán y Contreras, 2012; Oros et al., 2014). Así la conducta de apego no estaría limitada únicamente a la infancia temprana, pues aunque con menor frecuencia en la adolescencia y adultez se activaría en estados de estrés, ansiedad 0 angustia (Balabanian et al., 2014; Bowlby, 1995; Feeney y Noller, 2001).

El apego en este periodo puede ser considerado como una organización global, única e integrada que surge de la reflexión sobre las experiencias relacionales previas, manteniéndose más o menos estable y siendo capaz de predecir el comportamiento del adolescente dentro y fuera de la familia (Oliva, 2011).

Considerando que durante la adolescencia aumentan la búsqueda de libertad y autonomía, lo cual conlleva un sinnúmero de discusiones entre adolescentes y sus cuidadores, éstos comienzan a distanciarse afectivamente de ellos, mostrando rechazo y disminuyendo demostraciones de cariño. No obstante, los 
padres siguen siendo las figuras primarias de apego, quienes al brindarle el apoyo y la disponibilidad necesaria podrán generar una base estable y segura para que éstos formen su personalidad, y a su vez puedan adquirir estrategias que fomenten su propia seguridad (Balabanian et al., 2014; Oliva, 2011), de esta forma cuando los adolescentes tienen seguridad de que sus padres se encuentran disponibles y se les muestra apoyo podrán explorar libremente (Oliva, 2004).

Una investigación en adolescentes y apego (Kovak y Sceery, 1988), plantea que cuando los pares evalúan a sujetos con apego seguro aprecian menores índices de ansiedad en comparación con grupos con apego inseguro, además de índices más bajos de hostilidad en el estilo evitativo. A su vez el reporte de los sujetos con apego seguro involucraba menos estrés. Mikulincer, Shaver y Pereg (2003 en Garrido-Rojas, 2006), plantean que en el estilo seguro existe baja ansiedad y evitación, seguridad en el apego, comodidad con la cercanía y con la interdependencia, y confianza en la búsqueda de apoyo y otros medios constructivos de afrontamiento al estrés.

Al revisar cual es la relación específica entre los estilos de apego y la salud mental, se tiene que los adolescentes que presentan un apego seguro tienden a ser más abiertos, autónomos y socialmente aceptados, presentan menos sintomatología depresiva, menos conductas delictivas $y$, en general, niveles más bajos de psicopatología (Allen et al., 1998; Allen, Porter, McFarland, McElhaney y Marsh, 2007; Scott Brown y Wright, 2003 en Camps-Pons et al., 2014) y por otro lado quienes se clasifican dentro de los apegos de tipo inseguro se caracterizan por presentar mayor sintomatología ansiosa y depresiva, dificultades en sus relaciones y adaptación, así como un mayor riesgo de presentar trastornos psicopatológicos (depresión, problemas de conducta, suicidio, dependencia emocional, etc.) (Allen, Hauser y Borman-Spurrell, 1996; Allen et al., 2007; Fonagy, et al., 1996; Keskin y Çam, 2010;
McLewin y Muller, 2006; Muller, Lemieux y Sicoli, 2001; Scott Brown y Wright, 2003; Soares y Dias, 2007 en Camps-Pons et al., 2014).

Las relaciones de apego inseguros desorganizados se han propuesto como un factor de vulnerabilidad que predispone a los niños a una desregulación de su respuesta emocional, tendencias suicidas y síntomas asociados a trastorno de personalidad limítrofe al momento de llegar a la adolescencia (Lyons-Ruth, Bureau, Holmes, Easterbrooks, y Brooks, 2012), y si se considera la alta prevalencia de conductas autolesivas en sujetos víctimas de maltrato 0 aquellos que vivencian experiencias traumáticas en sus infancias, en conjunto con la clara afectación en torno a sus sistema de apego (Lyons-Ruth, 2010) es relevante poder abordar estas dimensiones en población no clínica, pues se podrán establecer factores de riesgo y protectores para futuras derivaciones 0 intervenciones en aquellos casos pesquisados.

Considerando lo anteriormente expuesto, a través de este estudio proponemos establecer la relación entre la presencia de autolesiones no suicidas con el tipo de apego en una muestra de adolescentes chilenos.

\section{MÉTODO}

El presente estudio es de tipo no experimental, transversal con aplicación de análisis estadísticos univariados $y$ bivariados. Realizamos un análisis exploratorio y descriptivo de los datos mediante el uso del paquete estadístico Statistical Package for the Social Sciences (IBM SPSS) versión 22. El tipo de muestreo utilizado fue no probabilístico por conveniencia.

\section{Instrumentos}

Los cuestionarios utilizados fueron: Escala elaborada ad hoc según los planteamientos del DSM 5 relacionados con la presencia de autolesiones no suicidas (American 
Psychiatric Association, 2014) para el cual realizamos una validación de contenido y juicio de expertos mediante coeficiente Kappa de Cohen (Cohen, Swerdlik y Smith,1992 en Cerda Lorca \& Villarroel Del P., 2008).

Para medir apego utilizamos dos escalas, el Relationship Questionnaire (RQ) del año 1991 de los autores Bartholomew y Horowitz versión validada en Chile (Gonzalez y Méndez, 2006) ajustando los puntajes de medición, el cual se caracteriza por una alta consistencia con diversos criterios. Dicho cuestionario está compuesto por cuatro párrafos que miden los estilos de apego seguro, preocupado, ansioso e indiferente, en el cual los sujetos indican en una escala entre uno y cinco en qué medida son descrito por cada prototipo. Además, se utilizó el Cuestionario de Apego en Adolescentes (AAQ por su nombre en inglés Adolescent Attachment Questionnaire). El AAQ, el cual fue creado en el año 1998 por West, Rose, Spreng, Sheldon-Keller y Adam, a partir de los planteamientos de Ainsworth y Bowlby en relación con el apego, derivándose teóricamente en tres escalas. Este cuestionario a diferencia de otros no entrega un índice de seguridad o inseguridad, sino que está diseñado para evaluar la percepción sobre la disponibilidad y responsividad de la figura de apego del adolescente, asociado a las dimensiones disponibilidad, cooperación en la relación y señales de enojo y angustia en su validación chilena (Morán et al., 2014).

\section{Participantes}

Participaron 186 adolescentes, con edades comprendidas entre los 14 y 18 años $(M=$ $15,8 ; \mathrm{DE}=1,1)$, de los cuales $80(43 \%)$ corresponden a hombres, y $106(57 \%)$ corresponden a mujeres. De los 186 adolescentes que cursan enseñanza media: 54 pertenecen a primero medio, 57 a segundo medio, 48 a tercero medio, y 27 a cuarto medio.
En cuanto al establecimiento educacional al que pertenecen los adolescentes parte de este estudio, es un establecimiento particular subvencionado gratuito con un índice de vulnerabilidad escolar de 65,8\% (Infante, Matus, Paulsen, Salazar, y Vizcarra, 2013). El mismo recibe a estudiantes de comunas caracterizadas por ser zonas de desarrollo agrícola de temporada, fuerte presencia de población rural, altos niveles de analfabetismo, y población con estudios básicos y medios incompletos.

\section{Procedimiento}

El estudio contó con la aprobación del Comité de Ética Institucional de la Universidad de Santiago de Chile. Antes de la administración de los cuestionarios, reunimos a todos los padres y tutores de los adolescentes participantes donde se le explicó y se les pidió firmar el consentimiento informado. Luego de contar con la autorización, los adolescentes fueron reunidos en grupos donde se les explicó y se les pidió firmar el asentimiento informado, indicando que en caso de detectar alguna situación de riesgo se entregaría una nómina al establecimiento educacional para tomar las medidas correspondientes relacionadas con la atención en salud mental.

\section{RESULTADOS}

Con respecto a la presencia de autolesiones no suicidas, de los 186 sujetos 164 reportan no haberse realizado autolesiones no suicidas (88.2\%), y 22 reportan haberse realizado autolesiones no suicidas (11.8\%). De los que reportan autolesiones no suicidas, $22.7 \%$ (5 casos) corresponde a hombres y $77.3 \%$ (17 casos) corresponden a mujeres.

Al considerar la edad de quienes reportan autolesiones $18.2 \%$ tienen 14 años; $36.3 \%$ tienen 15 años; $27.3 \%$ tienen 16 años; $13.6 \%$ tienen 17 años; y $4.5 \%$ tienen 18 años. 
Si se consideran los resultados del cuestionario de relaciones que mide los cuatro prototipos de apego se obtiene lo que se observa a continuación: Apego seguro
$38.2 \%$; apego evitativo 20.8\%; apego ambivalente 20.2\%; apego desorganizado $20.8 \%$.

TABLA 1.

Distribución de estilos de apego según escala $R Q$.

\begin{tabular}{llccc}
\hline & & $f$ & $\%$ & $\%$ válido \\
\hline Válido & Apego seguro & 68 & 36,6 & 38,2 \\
& Apego evitativo & 37 & 19,9 & 20,8 \\
& Apego ambivalente & 36 & 19,4 & 20,2 \\
& Apego desorganizado & 37 & 19,9 & 20,8 \\
& Total & 178 & 95,7 & 100,0 \\
Perdidos & Sistema & 8 & 4,3 & \\
Total & & 186 & 100,0 & \\
\hline
\end{tabular}

Con respecto al Cuestionario de Apego en Adolescentes la Tabla 2 muestra los resultados de las tres sub-escalas (disponibilidad, cooperación; y señales de enojo y angustia):

TABLA 2.

Resultados percepción de las sub escalas cuestionario de apego en adolescentes.

\begin{tabular}{cccccccccc}
\hline & \multicolumn{3}{c}{ Disponibilidad } & \multicolumn{3}{c}{ Cooperación } & \multicolumn{3}{c}{ Señales de enojo y angustia } \\
\cline { 2 - 10 } & Baja & Media & Alta & Baja & Media & Alta & Baja & Media & Alta \\
\hline $\mathrm{f}$ & 2 & 22 & 155 & 2 & 15 & 168 & 59 & 88 & 38 \\
$\%$ & 1,1 & 12,3 & 86,6 & 1,1 & 8,1 & 90,8 & 31,9 & 47,6 & 20,5 \\
\hline
\end{tabular}

Para realizar las comparaciones sobre el estilo de apego en aquellos sujetos que reportan autolesiones no suicidas y los que no, se llevó a cabo la prueba estadística de Chi cuadrado, siendo una prueba estadística para evaluar hipótesis acerca de la relación entre dos variables categóricas (Hernandez,
Fernandez y Baptista, 2010). Al comparar los estilos de apego reportados en el cuestionario de relación se observa que aquellos sujetos que reportan autolesiones presentan mayoritariamente estilos de apego de la línea ambivalente (33.3\%) y desorganizado (42.8\%). (Tabla 3).

TABLA 3.

Tabla de comparación autolesiones y tipo de apego, utilizando tablas cruzadas.

\begin{tabular}{llccccc}
\hline & \multicolumn{4}{c}{ Cuestionario de relaciones } & \multirow{2}{*}{ Total } \\
\cline { 3 - 6 } & & $\begin{array}{c}\text { Apego } \\
\text { seguro }\end{array}$ & $\begin{array}{c}\text { Apego } \\
\text { evitativo }\end{array}$ & $\begin{array}{c}\text { Apego } \\
\text { ambivalente }\end{array}$ & $\begin{array}{c}\text { Apego } \\
\text { desorganizado }\end{array}$ \\
\hline \multirow{2}{*}{ Autolesiones } & Sin autolesiones & 64 & 36 & 29 & 28 & 157 \\
Total & Autolesiones & 4 & 1 & 7 & 9 & 21 \\
& & 68 & 37 & 36 & 37 & 178
\end{tabular}

TABLA 4.

Pruebas de chi cuadrado para tabla cruzada entre quienes se autolesionan y su tipo de apego.

\begin{tabular}{lccc}
\hline & Valor & gl & Sig. asintótica (2 caras) \\
\hline Chi-cuadrado de Pearson & 12,830 a & 3 &, 005 \\
Razón de verosimilitud & 13,042 & 3 &, 005 \\
Asociación lineal por lineal & 10,162 & 1 &, 001 \\
N de casos válidos & 178 & & \\
\hline a. 3 casillas (37,5\%) han esperado un recuento menor que 5. El recuento mínimo esperado es 4,25.
\end{tabular}


Existe una asociación estadísticamente significativa $(p \leq .005)$ entre quienes se autolesionan y los que no, respecto al tipo de apego que reportan $\left(X^{2}=12,83\right)$.

En relación con las tres dimensiones que se observan en el cuestionario de apego de adolescentes, tanto para para la variable disponibilidad, como para la variable cooperación no existen asociación estadísticamente significativa. Sin embargo, para la variable señales de enojo y angustia si existe asociación estadísticamente significativa.

TABLA 5.

Tabla de comparación autolesiones y señales de enojo y angustia utilizando tablas cruzadas.

\begin{tabular}{llcccc}
\hline & & \multicolumn{3}{c}{ Señales de enojo y angustia } & \multirow{2}{*}{ Total } \\
& & Bajas & Media & Altas & \\
\hline \multirow{2}{*}{ Autolesiones } & Sin autolesiones & 58 & 77 & 28 & 163 \\
\cline { 2 - 5 } Total & Autolesiones & 1 & 11 & 10 & 22 \\
\hline
\end{tabular}

Quienes reportan autolesiones presentan señales medias $(50 \%)$ y altas $(45.4 \%)$ de enojo y angustia frente al cuidador.
Existe una asociación estadísticamente significativa $p \leq .001$ entre quienes se autolesionan y los que no respecto a las señales de enojo y angustia $\left(X^{2}=13,43\right)$.

TABLA 6.

Pruebas de chi cuadrado para tabla cruzada de quienes se autolesionan y el puntaje de las señales de enojo y angustia hacia su cuidador.

\begin{tabular}{lccc}
\hline & Valor & gl & Sig. asintótica (2 caras) \\
\hline Chi-cuadrado de Pearson & 13,431 a & 2 &, 001 \\
Razón de verosimilitud & 14,712 & 2 &, 001 \\
Asociación lineal por lineal & 13,262 & 1 &, 000 \\
N de casos válidos & 185 & & \\
\hline a. 1 casillas (16,7\%) han esperado un recuento menor que 5. El recuento mínimo esperado es 4,52.
\end{tabular}

\section{DISCUSIÓN}

Si se considera la prevalencia estimada respecto a la presencia de autolesiones en población adolescente no clínica, la cual va entre un 12 y un $28 \%$ (Frías Ibáñez et al., 2012; Washburn et al., 2012) y se compara con los resultados obtenidos en el presente estudio $11.8 \%$, se podría plantear que está cerca de aquel grupo estudiado.

En cuanto a la distribución por sexo de quienes reportan autolesiones (mujeres $77.3 \%$; hombres $22.7 \%$ ), considerando que la muestra en general es mayoritariamente femenina, de este estudio se obtienen resultados en la misma línea de estudios de prevalencia de autolesiones, la cual es mayor en mujeres que en hombres (Hawton et al., 2002; Patton et al., 2007; Morey et al.,
2008; O'Connor et al., 2009; Shin et., 2009; Landstedt y Gillander, 2011, en Frías Ibáñez et al., 2012).

En cuanto a estilo de apego se plantea que existen proporciones en que dos de cada tres personas presentan apego seguro, versus uno cada tres personas presentan apego de tipo inseguro (Camps-Pons et al., 2014), sin embargo, en la población evaluada se da una proporción contraria en que un $38,2 \%$ reporta apego de tipo seguro y un $61,8 \%$ presenta apego inseguro $(20,8 \%$ apego evitativo; $20,2 \%$ apego ambivalente; $20,8 \%$ apego desorganizado). Elementos que darían cuenta de que es un grupo que presenta mayor vulnerabilidad en cuanto a sus vínculos de apego. En cuanto a esto, se entiende que los vínculos de apego surgen asociados a atributos relacionales $y$ 
experiencias interpersonales, por lo que grupos como el estudiado, estarían más expuestos a situaciones adversas como abandono, maltrato, negligencia, etc. (Hessels et al., 2018).

La mayor proporción de apego inseguro, se podría deber a que el cuestionario $R Q$ que fue aplicado para medir esa variable, se ha utilizado principalmente en población adulta (Martínez y Santelices, 2005), y escasamente en población adolescente (Gonzalez y Mendez, 2006), por lo que las características dela adolescencia asociadas a búsqueda de identidad e impulsividad, así como elementos propios del desarrollo de la personalidad podría verse reflejado en las respuestas más asociadas a la forma en que ellos se ven. (Florenzano, 1998).

El cuestionario plantea características generales en torno a la forma de relacionarse con otros, elemento clave considerando que las principales relaciones de los adolescentes son con su grupo de pares, las cuales pueden ser muy fluctuantes (Oliva, 2011), razón por la cual es necesario el contraste con el otro cuestionario que mide específicamente dimensiones del apego considerando al cuidador principal desde la infancia en adelante (Morán et al., 2014).

A lo anterior se suma la percepción que poseen los adolescentes respecto de su figura primaria de apego en las variables que mide el cuestionario de apego adolescente (Morán et al., 2014), donde un $98.9 \%$ de la muestra reporta que observan que la disponibilidad de su figura de apego es alta 0 media; un $98.9 \%$ reporta que la cooperación es alta o media; en contraste con el $68.1 \%$ que reporta altas y medias señales de enojo y angustia respecto de su figura de apego.

Al momento de contrastar los resultados obtenidos en los estilos de apego en aquellos sujetos que reportan haberse infligido autolesiones no suicidas mayoritariamente presenta apego de tipo inseguro y señales media a alta en la subescala señales de enojo y angustia hacia la figura de apego . Dichos elementos tienen relación con lo planteado en distintos estudios en que se plantea que los estilos de apego inseguros y desorganizados son un factor de vulnerabilidad que predispone a los niños a una desregulación de su respuesta emocional, tendencias suicidas y síntomas asociados a trastorno de personalidad limítrofe al momento de llegar a la adolescencia (Lyons-Ruth et al., 2012), por lo tanto las autolesiones podrían ser utilizadas como una forma de regulación emocional (Klonsky, 2007, 2009).

En ese contexto cabe señalar lo que plantean (Tatnell, Hasking, y Newman, 2017) referido a que la capacidad reguladora de las emociones se desarrollaría en el contexto de las relaciones de apego, y podría estar relacionada con la calidad de éstas relaciones, pudiendo aumentar el riesgo de presentar autolesiones como resultado de dificultades en el apego. Los elementos presentados anteriormente tienen relación con la propuesta de Hallab y Covic (2010), quienes plantean que aquellos adolescentes que se autolesionan tienden a tener vínculos de apego menos seguro con los padres y sus cuidadores, en contraste con quienes no se autolesionan.

Si se considera que aquellas personas que se autolesionan son más propensas a fuertes y frecuentes emociones negativas, $y$ a su vez reportan acceso limitado a otras estrategias más adaptativas para sentirse mejor (Hollander, 2017; Victor y Klonsky, 2014), tiene completa coherencia con que aquellos sujetos que reportan autolesiones presentan significativamente altas y medias señales de enojo y angustia hacia las figuras de apego, dando cuenta que dichas emociones tendrían relación con su conducta autolítica.

Lo anterior se suma a que aquellos individuos que se autolesionan informan que carecen de estrategias alternativas para regular sus emociones (Hollander, 2017; 
Victor y Klonsky, 2014). Por lo tanto, las experiencias tempranas y las capacidades de regulación actuales aumentan el riesgo de cometer autolesiones (Tatnell et al., 2017).

Respecto a la forma en que expresan sus emociones las personas con estilo de apego ambivalente, éstos tienden a intensificar su expresión emocional (Mikulincer y Shaver, 2007 en Espinosa, 2015), y por lo tanto una de forma de intensificar su expresión emocional podría ser mediante la presencia de autolesiones. Dichos elementos podrían relacionarse con algunos aspectos típicos de los trastornos de personalidad en la adultez, en que existe una exageración de síntomas $y$ afectos negativos, al igual que una alta preocupación por las dificultades vividas en las relaciones interpersonales, junto con el modelo interno de cuidadores incompetentes 0 inconsistentes en su disponibilidad, lo que puede traer sentimientos de enojo hacia los cuidadores (Espinosa, 2015; Morán et al., 2014). Tal como queda planteado en los resultados, en que aquellos que se autolesionan presentan mayoritariamente apego de tipo ambivalente, y a su vez medios y altos grados de señales de enojo y angustia respecto de sus cuidadores.

Una de las conclusiones principales del presente estudio es que es de gran utilidad abordar el constructo del apego en población adolescente, como elemento clave al momento de comprender la presencia de sintomatología de todo orden como ansiedad, estrés, depresión, etc., asi como la presencia de diversas conductas negativas como evitación, aislamiento, enojo, desregulación emocional, las cuales mal manejadas pueden llevar a actuar de forma arriesgada aumentando la posibilidad de autolesionarse, y por lo tanto aumentar el riesgo de suicidio.

Por lo tanto, evaluar dichos elementos permitirán abordar la intervención desde un enfoque que mejore las relaciones de apego, en que el terapeuta se vuelve una base segura y consistente, lo cual podría mejorar las respuestas de los pacientes en cuanto a las autolesiones (Teague-Palmieri y Gutierrez, 2016). Los psicoterapeutas funcionan como figuras de apego sustitutas en aquellos pacientes que buscan protección respecto a una amenaza percibida por el self, proporcionando una base segura para la exploración de experiencias anteriores (Crittenden, 2000).

Lo anterior se suma a la premisa de que las figuras de apego posibilitan la construcción de un modelo de mundo y de sí mismo en función del desarrollo cognitivo y afectivo del sujeto, que permite actuar, comprender la realidad y anticipar el futuro en relación al establecimiento de metas. Dichos modelos de realidad son estructuras cognitivas que forman modelos representacionales del mundo, siendo la única forma que tiene el individuo para establecer relación con éste. Por lo que las construcciones autorreferenciales recíprocas con la imagen de la persona significativa permiten estabilizar y desarrollar la dinámica relacional. Así en la terapia se pueden proporcionar herramientas de análisis y autoobservación para lograr un reordenamiento gradual de la experiencia personal, guiando el proceso de reorganización (Guidano, 1994).

En cuanto a las limitaciones del estudio se encuentra el tamaño de la muestra, pues se requieren de muestras mucho más grandes para aumentar el poder estadístico, así como el uso de técnicas multivariadas para complementar la información. Otro elemento que puede ser considerado como una limitación tiene que ver con la forma de aplicar los cuestionarios, pues al ser de manera masiva existe el riesgo de que los adolescentes respondan rápido sin una lectura comprensiva de los ítems, así como que entreguen información limitada por la poca cercanía del evaluador, lo cual puede quedar demostrado en el bajo reporte de vivencias traumáticas, las cuales por su contenido pueden limitar la expresión de los adolescentes en una situación de evaluación 
masiva. Otro elemento que se considera relevante es haber incorporado variables para complementar la información como por ejemplo elementos asociados a regulación emocional, sintomatología psiquiátrica, así como elementos asociados a trastorno de personalidad de tipo limítrofe.

Surge como propuesta la posibilidad de comparar los resultados obtenidos en la presente investigación con los mismos datos en población clínica y diferenciando entre el tipo de autolesión, de manera de contrastar aquellos elementos que plantean que existe una mayor prevalencia de autolesiones en ciertos cuadros psicopatológicos (Frías Ibáñez et al., 2012; Washburn et al., 2012), así como la predominancia de estilos de apego desorganizados, ambivalentes y evitativos lo cual concuerda con que dichos estilos de apego se caracterizan por presentar mayor sintomatología ansiosa y depresiva, dificultades en sus relaciones y adaptación, así como un mayor riesgo de presentar trastornos psicopatológicos (depresión, problemas de conducta, suicidio, dependencia emocional, etc.) (Allen, Hauser y Borman-Spurrell, 1996; Allen et al., 2007; Fonagy, et al., 1996; Keskin y Çam, 2010; McLewin y Muller, 2006; Muller, Lemieux y Sicoli, 2001; Scott Brown y Wright, 2003; Soares y Dias, 2007 en Camps-Pons et al., 2014).

\section{REFERENCIAS}

American Psychiatric Association. (2014). Manual Diagnóstico y Estadístico de los Trastornos Mentales: DSM-5. Madrid, España: Editorial Médica Panamericana.

Baetens, I., Claes, L., Onghena, P., Grietens, $\mathrm{H}$., Van Leeuwen, K., Pieters, C., ... Griffith, J. W. (2014). Non-suicidal selfinjury in adolescence: A longitudinal study of the relationship between NSSI, psychological distress and perceived parenting. Journal of Adolescence, $37(6)$, 817-826. https://doi.org/10.1016/j.adolescence.2
014.05.010

Balabanian, C., Lemos, V., \& Vargas, J. (2014). Estudio psicométrico del Cuestionario de Apego Parental de Kenny en adolescentes argentinos. Acta Psiquiátrica y Psicológica de América Latina, 60(4), 227-235.

Bowlby, J. (1976). El Apego y la Pérdida, Vol. 2: La Separación Afectiva. Barcelona, España: Paidós.

Bowlby, J. (1979). Vínculos Afectivos: Formación, Desarrollo y Pérdida. Madrid, España: Morata.

Bowlby, J. (1995). Una base segura. Barcelona, España: Paidós.

Camps-Pons, S., Castillo-Garayoa, J. A., \& Cifre, I. (2014). Apego y psicopatología en adolescentes y jóvenes que han sufrido maltrato: implicaciones clínicas. Clinica y Salud, 25(1), 67-74. https://doi.org/10.1016/S11305274(14)70028-3.

Cassidy, J., \& Shaver, P. (2008). Handbook of Attachment. Theory, Research, and Clinical Applications. New York, Estados Unidos: The Gilford Press.

Cerda Lorca, J., \& Villarroel Del P., L. (2008). Evaluación de la concordancia interobservador en investigación pediátrica: Coeficiente de Kappa. Revista Chilena de Pediatria, 79(1), 54-58. https://doi.org/10.4067/S037041062008000100008.

Crittenden, P. (2000). Moldear la arcilla. El proceso de construcción del self y su relación con la psicoterapia. Psicoterapia, 41, 67-82.

Crittenden, P. (2006) A dynamic-maturational model of attachment. Australian and New Zealand Journal of Family Therapy, 27,105-116.

Crowell, S. E., Beauchaine, T. P., \& Linehan, M. M. (2009). A biosocial developmental model of borderline personality: Elaborating and extending linehan's theory. Psychological bulletin, 135(3), 495-510.

Espinosa, C. (2015). Rol de la Teoría de Apego como Factor de Cambio en la Gestión Terapéutica desde el Enfoque 
Constructivista Cognitivo. Tesis para optar al grado de magister. Facultad de Ciencias Sociales, Escuela de Postgrado, Universidad de Chile, Santiago, Chile.

Favazza, A. R. (1996). Bodies Under Siege : Self-mutilation And Body Modification In Culture and Psychiatry. Baltimore, Estados Unidos: Johns Hopkins University Press.

Feeney \& Noller. (2001). Apego Adulto. Bilbao, España: Desclée de brouwe

Florenzano, R. (1998). El Adolescente Y Sus Conductas De Riesgo. Santiago, Chile: Ediciones Universidad Católica de Chile.

Frías Ibáñez, Á., Vázquez Costa, M., Del Real Peña, A., Sánchez del Castillo, C., \& Giné Servén, E. (2012). Conducta autolesiva en adolescentes: prevalencia, factores de riesgo y tratamiento. Cuadernos de Medicina Psicosomática y Psiquiatria de Enlace, 103, 33-48.

Gandhi, A., Luyckx, K., Molenberghs, G., Baetens, I., Goossens, L., Maitra, S., \& Claes, L. (2019). Maternal and peer attachment, identity formation, and nonsuicidal self-injury: a longitudinal mediation study. Child and Adolescent Psychiatry And Mental Health, 13(1), 7.

Garrido-Rojas, L. (2006). Apego, emoción y regulación emocional. Implicaciones para la salud. Revista Latinoamericana de Psicologia, 38(3), 493-507.

Gonzalez, L., \& Mendez, L. (2006). Relación entre autoestima, depresión y apego en adolescentes urbanos de la comuna de Concepción, Chile. Terapia Psicológica, 24(1), 5-14.

Gratz, K. L. (2003). Risk Factors for and Functions of Deliberate Self-Harm: An Empirical and Conceptual Review. Clinical Psychology: Science and Practice, 10(2), 192-205. https://doi.org/10.1093/clipsy/bpg022

Guidano, V. F. (1994). El Sí Mismo en Proceso: Hacia una Terapia Cognitiva Posracionalista. Barcelona, España: Paidós.
Guzmán, M., \& Contreras, P. (2012). Estilos de apego en relaciones de pareja y su asociación con la satisfacción marital. Psykhe (Santiago), 21, 69-82. https://doi.org/10.4067/S071822282012000100005

Hallab, L., \& Covic, T. (2010). Deliberate selfharm: the interplay between attachment and stress. Behaviour Change, 27(02), 93-103. https://doi.org/10.1375/bech.27.2.93

Hawton, K., \& Harriss, L. (2007). Deliberate self-harm in young people: Characteristics and subsequent mortality in a 20-year cohort of patients presenting to hospital [CME].The Journal of Clinical Psychiatry, 68(10), 1574-1583.

https://doi.org/10.4088/jcp.v68n1017

Hawton, K., Rodham, K., Evans, E., \& Weatherall, R. (2002). Deliberate self harm in adolescents: self report survey in schools in England. BMJ (Clinical Research Ed.), 325(7374), 1207-1211.

Hernandez, R., Fernandez, C., \& Baptista, M. (2010). Metodología de la investigación. $5^{\circ}$ Edición. México: McGraw Hill Educación.

Hessels, C. J., Laceulle, O. M., van Aken, M. A. G., Resch, F., \& Kaess, M. (2018). Differentiating BPD in adolescents with NSSI disorder: the role of adverse childhood experiences and current social relationships. Borderline Personality Disorder and Emotion Dysregulation, 5(1), 1-11. https://doi.org/10.1186/s40479-0180097-5

Hollander, M. (2017). Helping Teens Who Cut, Second Edition: Using DBT® Skills to End Self-Injury.New York, Estados Unidos: The Gilford Press.

Infante, M., Matus, C., Paulsen, A., Salazar, A., \& Vizcarra, R. (2013). Narrando la vulnerabilidad escolar: Perfomatividad, espacio y territorio. Literatura $y$ Lingüística, 28, 281-308. https://doi.org/10.4067/S071658112013000100014

Klonsky, E. D. (2007). The functions of 
deliberate self-injury: A review of the evidence. Clinical Psychology Review,27, 226-239. https://doi.org/10.1016/j.cpr.2006.08.00 2

Klonsky, E. D. (2009). The functions of selfinjury in young adults who cut themselves: Clarifying the evidence for affect-regulation. Psychiatry Research, 166(2-3), 260-268. https://doi.org/10.1016/j.psychres.2008. 02.008

Kovak, R., \& Sceery, A. (1988). Attachment in late adolescence: working models, affect regulation and representations of self and others. Child Development, 59, 135-146.

Kristic, A. (2014). Patrones de apego y representaciones parentales en díadas con niños preescolares entre 2 y 5 años, de ambos sexos, que presentan sindrome de prader-will. Tesis de magister, Facultad de Ciencias Sociales. Universidad de Chile.Santiago. Chile

Lecannelier, F. (2009). Apego e Intersubjetividad. Influencia de los Vínculos Tempranos en el Desarrollo Humano y la Salud Mental. Segunda parte: La Teoría del Apego. Santiago, Chile: LOM Ediciones.

Lyons-Ruth, K. (2010). Repercusiones clínicas de los trastornos de las relaciones de apego desde la infancia a la adolescencia. Clinica e Investigación Relacional, 4(2), 340-356.

Lyons-Ruth, K., Bureau, J. F., Holmes, B., Easterbrooks, A., \& Brooks, N. H. (2012). Borderline symptoms and suicidality/self-injury in late adolescence: Prospectively observed relationship correlates in infancy and childhood. Psychiatry Research, 206(23), 273-281. doi.org/10.1016/j.psychres.2012.09.030

Main, M., \& Solomon, J. (1990). Procedures for identifying infants as disorganized/disoriented during the Ainsworth Strange Situation. En M.T.
Greenberg, D. Cicchetti, E. M. Cummings (Eds.). Attachment in The Preschool Years: Theory, Research, and Intervention (pp. 121-160). Chicago, The University of Chcago Press.

Martínez, C., \& Santelices, M.P.. (2005). Evaluación del Apego en el Adulto : Una Revisión. Psykhe, 14(1), 181-191. https://doi.org/10.4067/S071822282005000100014.

Mollá, L., Vila, S. B., Treen, D., López, J., Sanz, N., Martín, L. M., ... Bulbena, A. (2015). Autolesiones no suicidas en adolescentes: Revisión de los tratamientos psicológicos. Revista de Psicopatologia y Psicologia Clinica, 20(1), 51-61. https://doi.org/10.5944/rppc.vol.1.num.1 .2015.14408.

Morán, J., Lecannelier, F., \& Rodríguez, J. (2014). Estudio de validación de un cuestionario de apego en adolescentes. Revista Chilena de Pediatria, 85(4), 437-442.

https://doi.org/10.4067/S037041062014000400005.

Nader A, Morales AM. Síndrome de automutilación en adolescents: Análisis comparativo de comorbilidad. Revista Chilena de Psiquiatría y Neurología de la Infancia y Adolescencia,19(2):21-28.

Nixon, M. K., Cloutier, P., \& Jansson, S. M. (2008). Nonsuicidal self-harm in youth: A population-based survey. Canadian Medical Association Journal, 178(3), 306-312.

https://doi.org/10.1503/cmaj.061693.

Nock, M. K. (2010). Self-Injury. Annual Review of Clinical Psychology, 6(1), 339-363.

https://doi.org/10.1146/annurev.clinpsy. 121208.131258.

Nock, M. K., Joiner, T. E., Gordon, K. H., Lloyd-Richardson, E., \& Prinstein, M. J. (2006). Non-suicidal self-injury among adolescents: Diagnostic correlates and relation to suicide attempts. Psychiatry Research, 144(1), 65-72. https://doi.org/10.1016/j.psychres.2006. 
05.010.

Oliva, A. (2004). Estado actual de la teoría del apego. Revista de Psiquiatría y Psicología Del Niño y Adolescente, 4(1), 65-81.

Oliva, A. (2011). Apego en la adolescencia. Attachment during adolescence. Acción Psicológica, $\quad 8(2), \quad$ 55-65. https://doi.org/10.1007/978-1-44191695-2_4.

Organización Mundial de la Salud-OMS-. (2015). Estrategia mundial para la salud de la mujer, el niño y el adolescente (2016-2030): desarrollo en la primera infancia: informe del Director General (No. A71/19 Rev. 1). Organización Mundial de la Salud.

Oros, J., Vargas, J., \& Krumm, G. (2014). Parentalidad y emociones positivas durante la niñez. Acta Psiquiátrica y Psicológica de Ameríca Latina, 60, 2535.

Ougrin, D., \& Boege, I. (2013). Brief report: The self harm questionnaire : A new tool designed to improve identi fi cation of self harm in adolescents. Journal of Adolescence, $36(1), 221225$. https://doi.org/10.1016/j.adolescence.2 012.09 .006

Plener, P. L., Schumacher, T. S., Munz, L. M., \& Groschwitz, R. C. (2015). The longitudinal course of non-suicidal selfinjury and deliberate self-harm: a systematic review of the literature. Borderline Personality Disorder and Emotion Dysregulation, 2(1), 2. https://doi.org/10.1186/s40479-0140024-3

Pompili, M., Goracci, A., Giordano, G., Erbuto, D., Girardi, P., Klonsky, E. D., \& Baldessarini, R. J. (2015). Modern psychopathologies or old diagnoses. Journal of Psychopathology, 21, 348353.

Rojas, C. (2016). Autocortes: Una corporización de lo no dicho.Tesis de pregrado. Facultad de ciencias sociales. Universidad de Chile. Santiago. Chile.

Schore, A. (1997). Early organization of the nonlinear right brain and development of a predisposition to psychiatric disorders. Development and Psychopathology, 9(4), 595-631. https://doi.org/10.1017/S095457949700 1363.

Schore, A. (2001). Effects of a secure attachment relationship on right brain development, affect regulation, and infant mental health. Infant Mental Health Journal, 22(2), 7-66.

Tatnell, R., Hasking, P., \& Newman, L. (2017). Multiple mediation modelling exploring relationships between specific aspects of attachment, emotion regulation, and non-suicidal self-injury. Australian Journal of Psychology. https://doi.org/10.1111/ajpy.12166.

Teague-Palmieri, E., \& Gutierrez, D. (2016). Healing together: family therapy resource and strategies for increasing attachment security in individuals engaging in nonsuicidal self-injury. The Family Journal, 24(2), 157-163. https://doi.org/10.1177/1066480716628 629.

Victor, S. E., \& Klonsky, E. D. (2014). Daily emotion in non-suicidal self-injury. Journal of Clinical Psychology, 70(4), 364-375.

https://doi.org/10.1002/jclp.22037.

Villarroel, J. G., Jerez, S. C., Angélica Montenegro, M. M., Montes, C. A., Igor, M. M., Silva, H. I., ... Clínico, P. (2013). Conductas autolesivas no suicidas en la práctica clínica. Primera parte: conceptualización y diagnóstico Non suicidal self injuries in clinical practice. Revista Chilena de Neuro-Psiquiatría, 51(1), 38-45. https://doi.org/10.4067/S071792272013000100006.

Washburn, J. J., Richardt, S. L., Styer, D. M., Gebhardt, M., Juzwin, K. R., Yourek, A., \& Aldridge, D. (2012). Psychotherapeutic approaches to nonsuicidal self-injury in adolescents. Child and Adolescent Psychiatry and Mental Health, 6(1), 14. https://doi.org/10.1186/1753-2000-6-14. 
West, M., Rose, M. S., Spreng, S., Sheldon-

Keller, A., \& Adam, K. (1998).

Adolescent attachment questionnaire: $A$

Todos los trabajos publicados en

brief assessment of attachment in

Revista Salud \& Sociedad (ISSNe:0718-7475)

están sujetos a una licencia Creative Commons

adolescence. Journal of Youth and

Adolescence, 27(5), 661-673.

https://doi.org/10.1023/A:10228912255

Reconocimiento 4.0 Internacional

42.

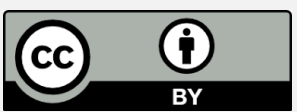

\section{RESUMO}

Objetivo: estabelecer a relação entre a presença de autolesões não suicidas com o tipo de apego em uma amostra de adolescentes chilenos. Método: Utilizou-se um questionário para avaliar autolesões não suicidas e dois questionários para medir 0 estilo de apego. Neste estudo não experimental, transversal e analítico, foi utilizado o teste do qui-quadrado para comparações de estilos de apego entre participantes que se automutam e aqueles que não fazem. Participaram 186 adolescentes, com idades entre 14 e 18 anos $(M=$ $15,8, \mathrm{DP}=1,1), 57 \%$ correspondem a mulheres. Resultados: $11 \%$ (22) dos sujeitos relataram autoagressão, $77,3 \%$ corresponderam a mulheres. Em indivíduos que relataram autoagressão, 80,9\% apresentavam algum tipo de apego inseguro (4,8\% evitativo, 33,3\% ambivalente e 42,8\% desorganizado). 95,4\% têm médias a altas pontuações nas subescalas de raiva e angústia em relação à figura do apego. Conclusões: Os estilos de apego inseguro e desorganizado são um fator de vulnerabilidade que predispõe os sujeitos à desregulação emocional. Adolescentes que relatam auto-mutilação tendem a ter um apego inseguro, em contraste com aqueles que ñ̃o se auto-prejudicam.

PALAVRAS-CHAVE: Auto-mutilação não suicida, apego, adolescentes.

\section{RESUMEN}

Objetivo: establecer la relación entre la presencia de autolesión no suicida con el tipo de apego en una muestra de adolescentes chilenos. Método: Utilizamos un cuestionario para evaluar autolesiones no suicidas y dos cuestionarios para medir estilo de apego. En este estudio no experimental, transversal y analítico, utilizamos prueba de Chi cuadrado para las comparaciones de los estilos de apego entre los participantes que se autolesionan y los que no. Participaron 186 adolescentes, con edades entre 14 y 18 años ( $M=$ $15.8, \mathrm{SD}=1.1$ ), el $57 \%$ corresponde a mujeres. Resultados: El $11 \%$ (22) de los sujetos reportaron autolesiones, el $77.3 \%$ correspondió a mujeres. En sujetos que reportaron autolesiones, el $80.9 \%$ tenía un tipo de apego inseguro $4.8 \%$ evitativo, 33.3\% de ambivalente y $42.8 \%$ desorganizado). El $95.4 \%$ tiene puntajes de medianos a altos en las subescalas de ira y angustia hacia la figura del apego. Conclusiones: los estilos de apego inseguros y desorganizados son un factor de vulnerabilidad que predispone a los sujetos a la desregulación emocional. Adolescentes que reportan autolesiones tienden a tener un apego inseguro, en contraste con aquellos que no se autolesionan.

PALABRAS CLAVE: Autolesiones no suicidas, apego, adolescentes. 